\title{
Infant with 3 beta hydroxysteroid dehydrogenase deficiency
}

\author{
K S D Kularatne ${ }^{1}$, D T W Arachchi ${ }^{2}$ \\ Sri Lanka Journal of Child Health, 2004; 33: 54-5
}

(Key words: 3-beta-hydroxysteroid-dehydrogenase deficiency, infant)

\section{Introduction}

Congenital adrenal hyperplasia $(\mathrm{CAH})$ is a group of autosomal recessive disorders of adrenal corticosteroid biosynthesis due to deficiency of one of 5 enzymes in the cholesterol to cortisol biosynthetic pathway. $90 \%$ of cases are due to deficiency of 21-hydroxylase while the remainder are caused by a lack of 11-hydroxylase or 3 beta hydroxysteroid dehydrogenase ${ }^{1}$. 3 beta hydroxysteroid dehydrogenase deficiency is associated with ambiguous genitalia in the newborn. There is potential ambiguity both in genotypic males and females in this condition. High levels of dihydroxy androsterone (DHA) and its peripheral conversion to stronger androgens result in variable clitoromegaly in females but are insufficient to fully masculinize a male infant resulting in variable hypospadias (often perineoscrotal form) with palpable testes. The defect in mineralocorticoid biosynthesis usually causes severe salt wasting. Mild defects are important causes of hirsutism presenting in young adult women and these late onset forms are commoner than the classical severe form. A structural defect of testis e.g. hypoplasia of Leydig cells, or more severe dysgenesis ${ }^{2}$ and partial deficiency of any of the enzymes en route to testosterone formation also results in genital ambiguity in a male (male pseudohermaphroditism) $)^{3}$.

\section{Case Report}

A premature 34 week old baby, with a birth weight of 1.47 Kg, was admitted to Special Care Baby Unit of General Hospital, Kalutara following an emergency caesarean section done for pregnancy induced hypertension on 3rd February 2002. Mother was 28 years old and this was her second child. Her first



(Received on 9 February 2004) child had died of leukaemia at the age of 3 months. Mother did not have any significant medical illnesses and her blood group was $\mathrm{O}$ positive. Her past obstetric history was uneventful. Baby had mild tachypnoea at onset with right basal shadowing in the chest $\mathrm{x}$-ray. The baby was started on intravenous (IV) penicillin and gen-tamicin pending blood culture and surface swab results.

On day two baby was active and pink with a regular respiratory rate of 55/min. Baby had good peripheral perfusion and regular femoral pulses. The heart rate was $120 /$ min and a grade $3 / 6$ systolic murmur was heard on $3^{\text {rd }}$ intercostal space at the left sternal border. Abdomen was not distended and no organomegaly was detected. Examination of external genitalia revealed an underdeveloped phallus, perineal urethra and a small scrotal sac. Small $(3 \mathrm{~mm})$ nodular structures were felt in the scrotal sacs.

The baby was started on gavage feeding which he tolerated well. Investigations were performed for further assessment of ambiguous genitalia. His serum electrolytes were as follows: Sodium $(\mathrm{Na}+)$ 138, potassium $(\mathrm{K}+) 6.01 \mathrm{meq} / 1$. Repeated result was $\mathrm{Na}+$ 138 and $\mathrm{K}+4.8 \mathrm{meq} / \mathrm{L}$. Ultrasound scan of the abdomen showed normal appearance with no evidence of tumour or haemorrhage of any viscera including adrenals. Kidney size and echogenicity was normal. Right kidney was 3 x $1.6 \mathrm{~cm}$ and left kidney $3.4 \times 1.5 \mathrm{~cm}$ in size.

On the 3rd day of life baby's condition rapidly deteriorated. Baby became lethargic, hypothermic and marked circulatory collapse was noted. Baby was cyanosed and apnoeic. This episode was successfully managed with intermittent positive pressure ventilation, volume replacement with normal saline boluses, followed by infusions of dextrose saline and a bolus dose of hydrocortisone. Baby made a good recovery after 30 minutes. Maintenance dose of hydrocortisone was not started as the endocrine studies were to be completed. At this stage salt losing adrenogenital syndrome was suspected. To combat 
any concurrent infection antibiotics were continued and blood cultures were repeated.

On the 4th day baby suddenly deteriorated again. Baby had a respiratory arrest and an episode of circulatory collapse. There was bradycardia and random blood sugar (RBS) was low. This episode was also successfully managed with cardiopulmonary resuscitation, IV hydrocortisone, boluses of normal saline and $10 \%$ dextrose. Further management included dextrose saline infusions and IV hydrocortisone. Subsequently therapy was changed over to oral hydrocortisone and fludrocortisone. Baby was discharged home after 5 weeks of hospital stay and subsequently reviewed in the clinic. Weight gain was satisfactory.

At the age of 3 months he was found to have an indirect inguinal hernia which was successfully repaired by the consultant surgeon under general anaesthesia.

Further investigations were as follows: Platelet count was $158 \times 10^{9} / \mathrm{L}$, white cell count $12.9 \times 10^{9} / \mathrm{L}$ (N50, L49, E01); serum electrolytes sodium $(\mathrm{Na}+)$ $129 \mathrm{meq} / \mathrm{l}$, potassium $(\mathrm{K}+) 5.6 \mathrm{meq} / \mathrm{l}$; blood group: A positive; blood cultures (two) sterile; baby's urinary electrolytes at this stage were $\mathrm{Na}+100 \mathrm{mmol} / \mathrm{l}$ (ref. range $1-15 \mathrm{mmol} / \mathrm{l}$ ) and $\mathrm{K}+$ was $2.5 \mathrm{mmol} / 1$. This reveals marked sodium loss in the urine. Chromosomal analysis done subsequently was 46XY. Seventeen hydroxyprogesterone level was normal.

\section{Discussion}

This baby has clinical features suggestive of deficiency of hydrocortisone, aldosterone and testosterone. Clinically, the presence of palpable gonad and negative buccal smear are in favour of male sex which was confirmed by the chromosomal analysis of $46 \mathrm{XY}$. Seventeen hydroxyprogesterone levels were within the normal range.

Two theoretical possibilities considered at this stage were, 3 beta hydroxylase dehydrogenase deficiency and 20,22 cholesterol desmolase deficiency. In 20,22 desmolase deficiency both genetic males and genetic females are phenotypically females and the condition is incompatible with life. In our case clinical features, biochemistry and chromosomes are all highly suggestive of 3 beta hydroxysteroid dehydrogenase deficiency. Enzyme assay would confirm the diagnosis.
This baby is doing well on oral hydrocortisone and fludrocortisone. Further management includes testosterone therapy at puberty. Human chorionic gonadotrophin stimulation test needs to be done to see the testosterone response before testosterone therapy. Adrenal lymphocytic infiltration and adrenocortical tumours have been reported very rarely with inadequate cortisol therapy in congenital adrenal hyperplasia ${ }^{4}$. Testicular lesions containing adrenal-like tissue can be found in some males with $\mathrm{CAH}$ with its function increased by corticotrophin and decreased by high dose of glucocorticoids ${ }^{5}$.

\section{References}

1. Lifford R J, Dear P R F. Intersex baby. British Journal of Hospital Medicine 1987; 29: 32.

2. Brown D M, Markland C, Dehner L P. Leydig cell hypoplasia, a cause of male pseudohermaphroditism. Journal of Clinical Endocrinology and Metabolism 1918; 46: 1-7.

3. Griffin J E, Wilson J D. Hereditary male pseudohermophroditism. Clinics in Obstetrics and Gynaecology 1978; 5: 457-79.

4. Merke D P, Bornstein S R, Braddock D. Adrenal lymphocytic infiltration and adrenocortical tumours in a patient with 21hydroxylase deficiency. The New England Journal of Medicine 1999; 340: 1121.

5. Ovarian adrenal like tissue in congenital adrenal hyperplasia. The New England Journal of Medicine 1998; 339: 853-4. 
